\title{
PREDICATIVITY AND CONSTRUCTIVE MATHEMATICS
}

\author{
LAURA CROSILLA
}

Keywords: Inductive definitions, Predicativity, Constructive Mathematics, Vicious Circle Principle, Invariance.

\section{INTRODUCTION}

Constructive mathematics is a form of mathematics which uses intuitionistic rather than classical logic. Different varieties of mathematics based on intuitionistic logic have been proposed over the years since Brouwer's inception of intuitionism. In the following, "constructive mathematics" denotes "Bishop-style" mathematics, the mathematics based on intuitionistic logic initiated by Errett Bishop in "Foundations of constructive analysis" [9]. ${ }^{1}$ Constructive mathematics has since witnessed substantial advances in analysis, topology and algebra. Starting from the 1970's, a number of formal systems have been proposed to codify or formalise this form of mathematics. Their aim was to isolate the principles underlying constructive mathematics' fundamental concepts, especially its concepts of set and function. Among these systems are Martin-Löf Type Theory and Constructive Set Theory. ${ }^{2}$

In this article, I consider one aspect in which constructive and classical foundations of mathematics differ. Constructive set and type theories diverge from standard classical set theories such as ZermeloFraenkel set theory in two distinct respects: they employ intuitionistic

\footnotetext{
Acknowledgments: This is a pre-print of an article to appear in Objects, Structures, and Logics, Springer. I would like to thank the editors of this volume, Stefano Boscolo, Gianluigi Olivieri and Claudio Ternullo for their determination in bringing the project of this volume to completion. I am grateful to Andrea Cantini and Øystein Linnebo for comments on an earlier version of this article. The research leading to this article has received funding from the European Union's Horizon 2020 research and innovation programme under the Marie Sklodowska-Curie grant agreement No 838445 .

${ }^{1}$ See [10] for an introduction.

${ }^{2}$ See e.g. $[43,48,2,45,7]$. Another approach to the foundations of constructive mathematics is Feferman's Explicit Mathematics, which has been studied especially in proof theory [27]. A very recent development is Homotopy Type Theory [71].
} 
rather than classical logic and they comply with a form of predicativity. Predicativity is my main objective, as I compare a prominent classical approach to predicativity with the form of predicativity that we find in constructive foundational systems. My focus is therefore not a comparison between constructive systems and standard classical foundations such as ZFC, rather a comparison between two distinct proposals for developing mathematics on the basis of a predicative concept of set. In so doing, I shed some light on the very notion of predicativity constructive systems contrive, which is not fully spelled out in the relevant literature.

In the following, I focus on a disagreement between standard classical and constructive approaches to predicativity. This regards the predicative status of so-called generalised inductive definitions. An inductive definition defines a set, say $X$, by: (i) identifying some initial elements of $X$; (ii) specifying new elements of $X$ in terms of elements already included in it and (iii) finally adding that nothing else belongs to X. Inductive definitions are clearly appealing from a constructive point of view, as they present a set as if it were constructed step-by-step from below. The metaphor of a step-wise generation of a set from below is also often employed to convey the notion of predicative definition of a set, i.e. of a definition that is not viciously circular. While salient inductive definitions are considered constructive, their predicative status is disputed. According to constructive approaches to predicativity, such as the one developed in Martin-Löf type theory, generalised inductive definitions are acceptable. They are, however, impredicative according to a well-known classical approach to predicativity. ${ }^{3}$

The remarkable feature of this disagreement is that constructive approaches to predicativity may be seen as more "generous" compared with standard classical approaches to predicativity. This fact is at first sight surprising, since we usually expect constructive foundations to be more restrictive than their classical counterparts. Constructive foundations are indeed substantially more restrictive than impredicative foundations such as ZFC, in the sense that they do not countenance impredicative and essentially non-constructive methods of proof. ${ }^{4}$ However, if

\footnotetext{
${ }^{3}$ As further clarified in section 4.1, the debate on the predicative status of inductive definitions has focused on generalised inductive definitions. In the following, unless otherwise stated, I omit the qualification "generalised".

${ }^{4}$ Arguably, from a different prespective, constructive systems are more flexible and less restrictive than traditional classical systems such as ZFC, as they allow for a variety of interpretations, including computational interpretations (see section 2). See also [10].
} 
we consider predicative approaches to foundations, the standard classical approach elaborated, for example, by Kreisel, Feferman and Schütte turns out to be more restrictive compared with the constructive one, and the key difference is its rejection of generalised inductive definitions. This observation can be made precise by employing fundamental results in ordinal analysis, a branch of proof theory. By carefully assigning ordinals to formal theories, proof theorists have devised means of comparing theories in terms of their "proof-theoretic strength". An outcome of that research is that constructive theories such as MartinLöf Type Theory and Constructive Zermelo Fraenkel set theory countenance systems which are proof-theoretically much stronger than classical theories that have been devised to codify (classical) predicativity. Crucially, these constructive systems have the resources to express generalised inductive definitions. ${ }^{5}$

This disagreement between classical and constructive forms of predicativity is pivotal for an understanding of predicativity and for an assessment of its significance within the foundations of mathematics. Inductive definitions play a substantial role within the contemporary constructive practice, as they are a fundamental component of constructive sets and type theories and also play a major role within constructive proof assistants such as Coq [70]. For this reason, an analysis of the foundational status of these definitions is timely and valuable.

Surprisingly, the relevant literature does not offer, as far as I know, a sharp delineation of the notion of constructive predicativity. More specifically, there is no detailed philosophical comparison between classical and constructive forms of predicativity nor an analysis of the above-mentioned disagreement between classical and constructive forms of predicativity. As (generalised) inductive definitions are considered a crucial component of constructive predicativity, an analysis of this disagreement between classical and constructive forms of predicativity is bound to shed light on the very notion of constructive predicativity and contribute to a more precise characterisation of this notion. For these reasons, the fundamental step into a philosophical investigation of constructive predicativity has to be an explication of the problem itself, i.e. of the disagreement between classical and constructive approaches to predicativity on the status of generalised inductive definitions.

In the first part of this article, I begin by offering some motivation for an enquiry in the predicative foundations of constructive mathematics, by looking at contemporary work at the intersection between mathematics and computer science. I then review the background notions

${ }^{5}$ See $[45,3,51,23,25]$. 
and spell out the above-mentioned disagreement between classical and constructive approaches to predicativity. In the second part of this article, I look at possible ways of defending the constructive predicativity of inductive definitions. Due to space constraints, I can only quickly sketch the main ideas. My proposal is to explore and further expand ideas on predicativity first put forth by Poincaré and Weyl at the turn of the $20^{t h}$ century, as they seem to offer a plausible route leading to the claim that inductive definitions are predicative from a constructive perspective. I also highlight the importance of clarifying whether the underlying logic, classical or intuitionistic, may have a role to play in assessing the predicative status of inductive definitions. A full assessment of the complex question of whether generalised inductive definitions are constructively predicatively justified but classically predicatively inadmissible will have to be postponed to another occasion. My hope at present is to generate some discussion on this important question and, more generally, on the very notion of constructive predicativity.

\section{Motivation: Constructive mathematics as Algorithmic MATHEMATICS}

One of constructive mathematics' most significant characteristics is that its theorems afford a computational or "algorithmic" interpretation: they can, at least in principle, run on a computer. Bishop's pioneer realisation that the exclusive use of intuitionistic logic could endow mathematical theorems with computational meaning has been vindicated in recent years. In fact, constructive mathematics and, especially, constructive type theory, have been fundamental source of inspiration for the theory and the applications of computer aided mathematics. One of the main instruments in this thriving area of research are proof assistants, i.e. computer software which is used interactively to formalize mathematical proofs. In recent times large and complex proofs of mathematical theorems, such as the Four Colour Theorem in graph theory and the Feit-Thompson Theorem in finite group theory have been implemented in such systems. ${ }^{6}$

Proof assistants are primarily used to completely formalize proofs and check their correctness. This is no trivial work, as a thorough formalization of a straightforward theorem requires not only to fill in all the gaps routinely left out in an informal proof and correct possible mistakes, but also formalize substantial portions of mathematics in view of all the background definitions and results the theorem depends on. It also involves subtle choices on how to best formalize

\footnotetext{
${ }^{6}$ See, for example, $[44,15,13,50,36,4,70]$.
} 
individual components of a proof. In addition to this "primary" application within mathematics the formalization of mathematical proofs has other uses, which are attracting renewed interest for this area of research. For example, proof assistants are also applied to verify the correctness of computer software. A further emerging area of research looks at utilizing proof assistants to "extract" computer programs from fully formalized proofs. Here constructive proofs have been the main focus so far, as we can make use of their interpretation as algorithms to produce real-life, working programs.

The extensive research on proof assistants, motivated as it is by a number of applications, is bound to have a considerable impact within mathematics. Mathematical proofs are becoming increasingly complex and large. Computer system that check the correctness of proofs are therefore likely to become a significant part of everyday mathematics. The hope is that computer systems could over time help us not only check existing large proofs, but also support us in finding new ones and develop effective proof strategies. Since constructive type theories (both predicative and impredicative) are at the heart of some of the most widely used proof assistants (e.g. Coq) these new developments may change significantly the perceived position of constructive mathematics within the mathematical community, granting it a more central role. For these reasons it is necessary that the philosopher of mathematics reflects on constructive mathematics and its philosophical motivations and compares it with the better-known classical practice.

Predicativity is a crucial component of foundational systems such as Martin-Löf type theory. The form of predicativity that we find in this theory combines the availability of a quite general form of inductive definitions (e.g. in the guise of so-called W types) with a strong form of Curry-Howard correspondence. The latter endows the logical constants with a direct computational meaning which is key to the theory's role as programming framework [44]. This interpretation of the logical constants, however, turns out to be incompatible with impredicativity, as demonstrated by Girard's paradox. ${ }^{7}$ Martin-Löf's way out of paradox was to abide to a form of predicativity while enriching the theory with powerful type constructing devices, i.e. W types and reflecting universes.

Predicativity is widely discussed from a technical point of view also within the Coq community. While the calculus of constructions (i.e. the type theory on which the Coq system is founded) features a strong

\footnotetext{
${ }^{7}$ See [34]. See also $[14,46]$ for analysis and [19] for philosophical reflections.
} 
form of impredicativity, recent versions of Coq have restricted this impredicativity so to gain more flexibility and ease compatibility with mainstream mathematics. Given the varieties of applications of proof assistants, it is important to allow for the possibility of adding assumptions that enable the formalization of different forms of mathematics, such as, for example, the axiom of choice or the principle of excluded middle, which are required to formalise standard classical mathematics. It is here that the notion of predicativity (in the form of syntactic constraints that block specific forms of impredicativity) has proved useful. ${ }^{8}$

As (generalised) inductive definitions are increasingly employed within the computer aided formalization of mathematics and are considered predicative there and within constructive mathematics, a clarification of their predicative status becomes particularly urgent. In this context, the disagreement between alternative approaches to predicativity that was mentioned in the Introduction becomes particularly significant. In the next section, I review the standard classical approach to predicativity which emerged from fundamental work in proof theory, before turning to the constructive case in subsequent sections.

\section{Predicativity given the natural numbers: the Classical APPROACH}

The notion of predicativity emerged at the beginning of the last century within Poincaré and Russell's analysis of the set-theoretic paradoxes. ${ }^{9}$ The analysis identified a form of vicious circularity as source of the paradoxes. This circularity is manifested in problematic impredicative definitions which attempt to define mathematical entities in a circular way, e.g. by specifying a "new" element of a collection by reference to all of that collection. Adherence to predicativity was therefore proposed as an instrument for avoiding vicious circularity in definitions and, in this way, stay clear of paradoxes. Russell introduced his well-known "Vicious-Circle Principle" (VCP), according to which a definition ought not to refer to (e.g. quantify over) a set containing the very entity it defines. ${ }^{10}$ Russell's ramified type theory was put forward

\footnotetext{
${ }^{8}$ In Coq there are two sorts (i.e. categories) of objects "Prop" and "Set". Both had impredicative features in early versions of the system, so that, for example, one could quantify over all Sets to define a new set. Recent versions, however, retain an impredicative "Prop" but abandon the impredicativity of "Set". These new restrictions are introduced to increase compatibility with classical mathematics [5].

${ }^{9}$ See, for example, $[54,55,56,62,61,63,57,58]$.

${ }^{10}$ Russell and Whitehead gave a number of renderings of the VCP. For example, " $n$ o totality can contain members defined in terms of itself" [63, p. 237] and "[...]
} 
to ensure full compliance with the VCP. ${ }^{11}$ The main idea of ramified type theory is to define sets (i.e. types) by introducing simultaneously two kinds of regimentation: type levels and orders. The latter regiment propositional functions so to ensure that properties defined by reference to the totality of properties of a given order belong to the next higher order. The interplay of these restrictions aims at avoiding the occurrence of the perceived problematic circularity in definitions. Avoiding vicious circularity in analysis was also Weyl's aim in "Das Kontinuum" [73], where a highly original and influential predicative treatment of analysis was undertaken without recourse to ramification. ${ }^{12}$

Following Poincaré and Russell, (im)predicativity is usually characterised as follows:

(i) a definition is impredicative if it defines an entity by reference to a totality to which the entity itself belongs; it is predicative otherwise.

(ii) a mathematical entity (e.g. a set) is impredicative if it can only be defined by an impredicative definition; it is predicative otherwise.

The qualification "only" in clause (ii) is important. This clause states that an entity is predicative provided that it affords a predicative definition. Since it is common for a mathematical entity to be defined in a number of equivalent ways, an entity is considered impredicative as long as no alternative predicative definition of it is available. Extensive work in mathematical logic in recent years has shown that many apparently impredicative notions in analysis can be reformulated so to afford predicative treatment. ${ }^{13}$ This work is complex, as one typically needs to re-frame one's definitions to avoid impredicativity. Sometimes this requires the redevelopment of a substantial portion of mathematics. Weyl's book "Das Kontinuum" [73] sets out a fundamental example in this respect, as it shows how to carry out large portions of analysis from a predicative point of view.

Of special interest in the present context are developments that took place from the 1950's, when prominent logicians undertook a precise formal analysis of predicativity. Only the most general points of that development are needed for the present discussion. ${ }^{14}$ Among these new

whatever in any way concerns all or any or some of a class must not be itself one of the members of a class" [64, p. 198]. See also [35] for an influential discussion, especially p. 454-5.

${ }^{11}$ See $[63,74]$.

${ }^{12}$ See Section 4.3 .1 for more on [73].

${ }^{13}$ See e.g. [28, 68, 69, 29, 32].

${ }^{14}$ Some of the most significant steps in that development are recalled in [30]. See also [21, 18]. 
developments, one course of thought brought to what is often termed "predicativity given the natural numbers" [30]. From a technical point of view, this may be seen as a continuation of both Russell's ramified type theory and Weyl's predicative analysis. It takes the VCP as the main guiding principle and further develops Russell's idea of ramification. It also takes the natural numbers as 'given', while introducing predicatively motivated constraints on subsets of the natural numbers, as Weyl did. The thought is that the natural numbers are unproblematic and safe, but sets of natural numbers need to be defined predicatively to avoid vicious circularity. Importantly, as in Weyl's 1918 booklet and in Russell's type theory, one uses classical logic throughout.

Notwithstanding these similarities, there is a significant difference with Weyl's and Russell's predicativism. The aim of the logical analysis of predicativity was not a predicative foundation of analysis, with the consequent abandonment of those parts of analysis that could not be rephrased in purely predicative terms. The main focus was rather a clarification -from the outside so to speak- of the limit of predicativity: how far can we reach if we take a predicativist stance? This question was approached along two main dimensions: (1) by using mathematical logic to determine the limit of predicativity and (2) by a case by case study of ordinary mathematics to assess which parts of it can be given predicative treatment. A further difference with Weyl is that the new attitude as well as the more refined logical instruments in the meantime available brought the logicians to go beyond Weyl's predicative analysis, by contemplating transfinite iterations of ramified comprehension along so-called predicative ordinals. Through fundamental contributions by Kreisel, Feferman and Schütte the "logical analysis of predicativity" gave rise to an exemplary chapter in proof theory, which culminated with the determination by Feferman and Schütte (independently) of the limit of predicativity by means of ordinal analysis. ${ }^{15}$

Ordinal analysis uses proof-theoretic techniques to assign ordinals to theories as a way of assessing and comparing their strength. The prooftheoretic analysis of predicativity of the 1960's made use of a transfinite hierarchy of subsystems of second order arithmetic with ramified comprehension (also called ramified analysis). The main idea is that each

\footnotetext{
15 See $[37,26,67,66]$. Note that this is not the only logical analysis of predicativity proposed in the 1950-60's. Another approach [38] made essential use of work in recursion theory and definability theory, and identified the predicatively definable sets of natural numbers with the so-called hyperarithmetical sets. Here work by Kleene, among others, provided fundamental insights and the necessary tools for the analysis. See [47] for the relevant notions, historical notes and references.
} 
system allows for a ramified form of comprehension, thus only "referring" to entities populating earlier stages of the hierarchy. This ensures that each level of the hierarchy is predicatively justified. Crucially, the hierarchy is indexed by ordinals and a substantial contribution of this analysis was a proposal on how far along the ordinals we may proceed without stepping into impredicativity. To this end, the notion of predicatively provable ordinal was introduced with the intention to capture the concept of an ordinal a predicativist would recognize. Roughly, predicatively provable ordinals can be defined "from below" through a bootstrapping process: one progresses along the ramified hierarchy to a theory indexed by an ordinal $\alpha$ only if one has already proved that $\alpha$ is an ordinal in a "previous" theory within the hierarchy. This hierarchy of formal systems then acted as canonical reference: one considers predicative any formal system which can be reduced to a system in that hierarchy (according to a formally specified notion of proof-theoretic reduction). The so-called limit of predicativity was then identified in terms of an ordinal known as $\Gamma_{0}$, the first non-predicatively provable ordinal. ${ }^{16}$

The logical analysis of predicativity therefore made a clear and precise proposal for a formal anlaysis of predicativity, employing state of the art logical machinery to extend Russell's and Weyl's work.

\section{Constructive PRedicativity and inductive Definitions}

A number of formal systems have been introduced over the years to formalise constructive mathematics. It is common to distinguish two kinds of systems: impredicative systems such as Intuitionistic Zermelo Fraenkel set theory and the Calculus of Constructions [33, 15], and predicative systems, such as Martin-Löf Type Theory and Constructive Zermelo Fraenkel set theory [43, 2]. While the latter theories are said to be predicative, the literature does not offer a sharp delineation of the relevant notion of predicativity, nor is there an authoritative analysis of this notion comparable to the insightful appraisal given over the years in the classical case, especially through the work of Feferman. ${ }^{17}$ There is agreement among constructive mathematicians on paradigmatic examples of impredicativity: as in the classical approach to predicativity

\footnotetext{
${ }^{16}$ Schütte's fundamental contribution to this analysis of predicativity is acknowledged by [31, p. 8-9] as follows: "[...] the determination by Schütte and me in the mid-1960s of $\Gamma_{0}$ as the upper bound for the ordinal of predicativity simply fell out of his ordinal analysis of the systems of ramified analysis translated into infinitary rules of inference when one added the condition of autonomy."

${ }^{17}$ For discussion see $[14,24,52,59]$.
} 
discussed above, the powerset of an infinite set is considered impredicative, and so is full second order arithmetic. Other forms of higher order quantification (e.g. over so-called "propositions" in type theory) are also considered impredicative. Moreover, there is overall agreement in the literature that some generalised inductive definitions are constructively predicatively justified. ${ }^{18}$ In fact, the acceptance of (at least some) generalised inductive definitions is often taken to be the main characteristic distinguishing the constructive from the classical approach to predicativity discussed in the previous Section. In fact, according to classical predicativity given the natural numbers generalised inductive definitions are impredicative, on the basis of proof-theoretic results.

In the following, I first review the notion of generalised inductive definition and then investigate why it is considered problematic from a classical predicativist perspective but may be considered unproblematic from a constructive point of view.

4.1. Inductive definitions. Inductive definitions were used in constructive mathematics from the very start as witnessed, for example, by Brouwer's constructive ordinals. In mathematical logic, inductive definitions gained particular relevance from the 1950's especially in recursion theory and in proof theory. ${ }^{19}$ A principal reason for the focus on inductive definitions in proof theory was Kreisel's hope that the study of formal theories for inductive definitions could clarify whether Spector's 1961's proof of consistency of second order arithmetic could be constructively justified. In fact, it turned out that such theories are not sufficiently strong to accomplish this task, as their proof-theoretic strength is strictly in between the strength of predicative theories (according to the $\Gamma_{0}$ analysis) and full second order arithmetic. However, the proof-theoretic investigation of theories of inductive definitions gave rise to crucial advances in ordinal analysis and was also key to the prooftheoretic study of prominent impredicative subsystems of second order arithmetic. $^{20}$

Today inductive definitions figure prominently in Martin-Löf type theory, for example in the form of well-founded trees, which are defined

\footnotetext{
${ }^{18}$ Note that while my focus in this note are intuitionistic theories, Lorenzen and Myhill have argued for a rather liberal notion of predicativity with respect to a quite general notion of constructivity (also in the context of theories with classical logic). See especially [41, 42]. See also [72]. For Martin-Löf type theory, see e.g. $[52,59]$.

${ }^{19}$ See the fundamental $[47,6]$.

${ }^{20}$ See [11]. The introduction gives an insight into the historical developments of ordinal analysis beyond predicativity. See Chapter 1 for background. See also $[31,46]$.
} 
by employing the so-called $\mathbf{W}$ type constructor. In the case of type theory, the combination of well-founded trees and universes (i.e. reflection principles) endow this theory with considerable proof-theoretic strength, well exceeding the strength of theories in the ramified hierarchy up to $\Gamma_{0}$. Martin-Löf type theory therefore includes systems whose proof-theoretic strength well exceeds the realm of predicativity given the natural numbers. ${ }^{21}$ Recent years have also seen frequent application of inductive definitions in constructive mathematics. For example, they have been successfully employed in formal topology $[65,16]$ to circumvent the ubiquitous use of the powerset operation. As already mentioned in Section 2, inductive definitions are also extensively used in the formalization of mathematics within theorem provers such as Coq. One reason for this is that inductive definitions offer a uniform way of characterising a number of type constructions, avoiding the proliferation of primitive types. For example, given a general scheme for inductive definitions, one can apply it to define the natural numbers, without assuming a primitive type of natural numbers. ${ }^{22}$

An inductive definition defines a set, say $X$, by identifying some initial elements of it and specifying all the remaining elements of $X$ in terms of elements already included in it. It may be helpful to see how one usually characterises inductive definitions from a standard set-theoretic perspective. Here an inductively defined set may be seen as the least fixed point of a monotone operator. For our purposes, it suffices to focus on the case of inductive definitions of sets of natural numbers. ${ }^{23}$ Let $\Gamma: P(\mathbb{N}) \rightarrow P(\mathbb{N}$ ) be an operator (or function) from the power set of the natural numbers to the power set of the natural numbers and let $X, Y \in P(\mathbb{N})$. We say that $\Gamma$ is monotone if:

$$
X \subseteq Y \rightarrow \Gamma(X) \subseteq \Gamma(Y) .
$$

$X$ is $\Gamma$-closed when

$$
\Gamma(X) \subseteq X
$$

For monotone $\Gamma$, it is easy to show that there is the smallest $\Gamma$-closed set, also called the fixed point of $\Gamma$ :

$$
I_{\Gamma}=\bigcap\{X: X \text { is } \Gamma \text {-closed }\} .
$$

The constructive appeal of inductive definitions is due to the fact that they can be thought of as constructing a set step-by-step and from below. This metaphor of a bottom-up construction can be made

\footnotetext{
${ }^{21}$ See e.g. $[51,60,52,23,25,59]$.

${ }^{22}$ See [24] for discussion and references.

${ }^{23}$ See the exposition in [11], Chapter 1.
} 
more precise by using the ordinals to index the stages of the least fixed point of a monotone operator. One starts from stage 0 and successively applies the operator $\Gamma$ to go from one stage to the next. More precisely, given $\Gamma$ as above, and $I_{\Gamma}$ its least fixed point, the $\alpha$-stage of $I_{\Gamma}$ is:

$$
I_{\Gamma}^{\alpha}=\Gamma\left(\bigcup_{\beta<\alpha} I_{\Gamma}^{\beta}\right)
$$

The crucial point is that, at the price of taking the classical ordinals as given, an inductively defined set can now be presented as the closure of a step-by-step process of generation, so that each stage is the result of applying the operator to a previously generated fragment of the set.

The reference above to the classical ordinals, however, is problematic from a constructive perspective. Another way of presenting inductive definitions may, however, be more appealing from a constructive perspective. This is in terms of a set of rules that specify the elements of an inductively defined set. Typically, one starts from some initial elements and then gives rules that yield new elements of a set from "previously constructed" elements of it. The least set closed under these rules is then the set inductively defined by them. ${ }^{24}$

The simplest example of inductive definition of an infinite set is the inductive definition of the set of natural numbers as the smallest set containing 0 and closed under the successor operation. One has the following introduction rules:

(1) 0 is a natural number,

(2) if $n$ is a natural number, then its successor, $\operatorname{suc}(n)$, is also a natural number.

Taking the natural numbers to be the smallest set satisfying these rules, amounts to adding the claim that nothing else is a natural number. The latter clause is expressed by the principle of mathematical induction, which is often formulated as an elimination rule complementing the introduction rules.

The example of the inductive definition of the natural numbers is here chosen for its simplicity. As already mentioned, both predicativity given the natural numbers and constructive predicativity take

\footnotetext{
${ }^{24}$ See [1]. Particularly appealing from a constructive point of view are deterministic rules. A rule is deterministic if for any conclusion $a$ there exists exactly one set of premises $X$ such that $a$ is a consequence of $X$ according to the rule.
} 
the natural numbers as unproblematic, as given, and introduce predicatively motivated constraints on subsets of the natural numbers. ${ }^{25}$ The inductive definitions that rise concerns from a standard (classical) predicativist perspective are those that go beyond the natural numbers, like, for example, the definition of the constructive ordinals. The latter can be defined by the following introduction rules:

(1) 0 is in $\mathcal{O}$,

(2) if $a$ is in $\mathcal{O}$, then $\operatorname{suc}(a)$ is in $\mathcal{O}$,

(3) if $f$ is a function from the natural numbers, $\mathbb{N}$, to $\mathcal{O}$ and for all $n$ in $\mathbb{N}, f(n)$ is in $\mathcal{O}$, then the supremum of the $f(n)$ is in $\mathcal{O}$.

While the inductive definition of the natural numbers is finitary, in the sense that each rule has only finitely many premises, the definition of the constructive ordinals is an example of infinitary inductive definition. Note also that the definition of the constructive ordinals builds on the definition of the natural numbers. One can further iterate this process and build a new inductive definition on the basis of the constructive ordinals, and so on. In this way, the so-called "higher tree classes" can be defined inductively. ${ }^{26}$ Generalised inductive definitions include definitions such as that of $\mathcal{O}$ and also countenance iterated inductive definitions.

The proof theory of inductive definitions has focused on formal theories that codify generalised inductive definitions. These formal theories extend Peano Arithmetic by introducing predicates for so-called positively definable operators. Here the positivity of the relevant predicates is required to ensure the monotonicity of the operators they define. ${ }^{27}$ Theories, based on intuitionistic logic (i.e. extensions of Heyting Arithmetic) have also been considered and have played a crucial role in the proof theoretic analysis. In practice, theories of inductive definitions have acted as systems of reference in the proof-theoretic analysis of inductive definitions, therefore playing a similar role in this context as systems of ramified analysis for the proof-theoretic analysis of predicativity. A well-known theory which formalises non-iterated inductive definitions goes under the name of $I D_{1}$. Stronger theories have been introduced to codify iterated inductive definitions. As already mentioned

\footnotetext{
${ }^{25}$ Note that while the forms of predicativity considered in this article take the natural numbers as unproblematic, this assumption is not gone unchallenged. Dummett, Nelson and Parsons have (independently) argued for the impredicativity of the principle of mathematical induction $[22,49,53]$. [49] develops a form of predicative arithmetic that substantially constrains mathematical induction, therefore giving rise to weak subsystems of Peano Arithmetic.

${ }^{26}$ See [11, p. 147].

${ }^{27}$ See $[11$, Chapter 1$]$.
} 
at the beginning of this section, the proof-theoretic analysis of theories of inductive definitions shows that their proof-theoretic strength exceeds that of predicative theories according to the $\Gamma_{0}$ analysis [11]. This is the case already for the theory $I D_{1}$, whose proof-theoretic ordinal, the so-called Bachmann-Howard ordinal, is much larger than $\Gamma_{0}$.

\subsection{The impredicativity of generalised inductive definitions.}

Generalised inductive definitions are considered impredicative according to the logical analysis of predicativity mentioned in Section 3. The worry is that in the build up of an inductive set we need to refer to the very set we are defining, thus contravening the VCP. In the terminology introduced in the previous section, the main difficulty lies in the claim that the inductively defined set we are defining is the least fixed point of the given inductive definition. This worry is particularly evident when we look at the standard set-theoretic presentation of inductive definitions. If we take the set-theoretic definition of the least fixed point of an inductive definition as the intersection of all $\Gamma$-closed subsets of $\mathbb{N}$, for some monotone operator $\Gamma$, then the difficulty is obvious: we define a subset of the natural numbers by reference to a collection of subsets of the natural numbers to which it belongs, against the VCP.

Arguably, one of the main benefits of the logical analysis of predicativity is that it has revealed that apparently impredicative notions of ordinary mathematics could after all be given a predicative treatment. As a consequence, a prima facie impredicativity could be eliminated. We could then explain a prima facie impredicativity as a by-product of its codification within a certain conceptual framework (e.g. set theory). One could hope that similar considerations could also be applied to the case of inductive definitions: while the set-theoretic framework strongly suggests the impredicativity of inductive definitions, a more careful analysis could perhaps offer a different verdict (at least in the case of the inductive definitions the constructivist cares about). For example, one could hope that an idea mentioned towards the end of the previous section could help defuse the impredicativity of inductive definitions. There we saw that the classical ordinals can be used to index the stages of the least fixed point of an inductive definition. The ordinals, in other terms, can help us stratify an inductively defined set so that at each step we refer only to "previously" constructed fragments of it. Borrowing the proof-theorist's terminology [11, p. 262-3], with the help of the ordinals, an inductive definition such as that of $\mathcal{O}$ can be expressed in such a way that it becomes locally predicative, i.e. it is predicative at each stage since, locally, we only refer to what has already been constructed, rather than to the whole set under 
construction. However, the difficulty with this strategy is that the ordinals we need to employ in order to index the stages of the inductive definition cannot be given a predicative justification that would satisfy the predicativist given the natural numbers. This is confirmed by the proof-theoretic analysis of theories of inductive definitions which are seen to exceed the proof-theoretic strength of predicative theories. In other terms, one could claim that we have local predicativity, but impredicativity as a whole.

Perhaps representing inductive definitions in terms of rules, with no explicit mention of the classical ordinals, could help explain away their impredicativity. The worry in this case is that the rules themselves may involve a circularity. In the case of generalised inductive definitions, in fact, the clause expressing the minimality of the inductive definition will have no restriction to prevent it from referring to the very set it inductively defines. ${ }^{28}$

These observations can be made fully precise through a careful prooftheoretic analysis of formal theories for inductive definitions. The main "argument" adduced for the impredicativity of inductive definitions, therefore, is the fact that the proof-theoretic strength of theories of inductive definitions exceeds the limit of predicativity given the natural numbers, captured by the ordinal $\Gamma_{0}$. In fact, there is no proof-theoretic reduction of theories of (generalised) inductive definitions to the systems of ramified analysis, since the former are proof-theoretically much stronger than the latter. As we saw above, the ramified hierarchy acts as canonical systems of reference for predicativity given the natural numbers. Therefore, the fact that the proof-theoretic strength of theories of inductive definitions exceeds the strength of the whole ramified hierarchy is taken as clear indication that generalised inductive definitions involve impredicativity.

4.3. Predicative after all? Although inductive definitions are considered impredicative according to predicativity given the natural numbers, they are usually considered constructive and predicative in the constructive literature. The term "constructive" is notoriously vague and is routinely applied to a variety of forms of mathematics, often very different from each other. It is thus perhaps not that surprising that the literature presents us with the claim that generalised inductive definitions are constructive, but impredicative. The availability of a set of rules for the generation of the elements of an inductively defined set

\footnotetext{
${ }^{28}$ A "miniature" argument along these lines can be carried out already in the case of the natural numbers to argue for the impredicativity of the induction principle. This will be discussed in section 4.3.2.
} 
is often considered key to the constructivity of inductive definitions. For example, when inductively defining an infinite set, one does so by means of fixed rules and in a uniform way: by employing some initial elements of the set and repeatedly applying a uniform procedure to obtain all the other elements of the set. Crucially, the induction principle associated with an inductive definition somehow mirrors the construction of the elements of the set. Hence, the proofs are also likewise structured. This latter point is rightly stressed by Sieg [11, Chapter 3, p. 147], when discussing the intuitionistic theory that formalises the construction over Heyting Arithmetic of the constructive ordinals. Sieg writes that this theory is constructively justified:

and by that I simply mean that the theory is based on intuitionistic logic, the objects in its intended model are exhibited or obtained by construction and the proofprocedures follow or parallel the construction of the objects.

While it is usually agreed that (at least some) generalised inductive definitions are constructive, it is their predicativity that is controversial $[26,11]$. For example, the inductive definition of $\mathcal{O}$ is considered constructively acceptable, but it is impredicative according to the prooftheoretic analysis of predicativity. To conclude this section, I sketch some options that a constructivist could consider to support the view that inductive definitions are after all not only constructive but also predicative.

4.3.1. Invariance. A first option is to link the contemporary discussion on inductive definitions directly to the original debate on predicativity. The idea is to focus on strong affinities that exist between the motivation offered today for the predicativity of inductive definitions and themes that pervade the original debate on predicativity at the beginning of the 20th century. I only consider two points, the role of infinity in this debate and the concept of set, even if an analysis of the relevant literature suggests further significant similarities.

In Section 3, I have presented a standard characterisation of predicativity in terms of lack of vicious circularity. Poincaré also offered another characterisation of predicativity in terms of a form of invariance, which seems more suitable to capture the phenomenon of inductive definitions. According to this new characterisation of predicativity, a 
predicatively defined set cannot be modified or disordered by an extension of the class of sets under consideration. ${ }^{29}$ This characterisation of predicativity relates to the one in terms of vicious circularity as follows: if we consider an impredicative definition (in the sense of circular), it would seem to have the effect of extending or enlarging a set under consideration. Let us see this with an example. Suppose we are given an impredicative definition of as set $X$ which refers to (e.g. universally quantifies over) a set $G$ to which $X$ belongs. For this definition to be meaningful, it would seem that we need first to fix the extent of the set $G$. But then $X$ would be a "new" element of $G$ which therefore extends or disorders $G$. Poincaré's requirement of invariance of mathematical definitions aims at avoiding definitionsof this kind: a predicative (in the sense of invariant) definition does not disorder a set by introducing new elements.

[73] proposed a detailed predicative foundation of analysis that bears important analogies with Poincaré's new characterisation of predicativity. Weyl's discussion, like Poincaré's, is characteristically bound up with his stark rejection of actual infinity in mathematics, advocating instead a potentialist view of infinity. In Weyl's case, this is further directly connected with his explicit rejection of arbitrary sets. The main idea, which is reminiscent of Poincaré, is that for a correct treatment of infinite sets, one needs predicative definitions. For Weyl this means that one defines an infinite set as the extension of some property or relation, which may be seen as describing a step-by-step process of formation of the set. ${ }^{30}$ In the case of analysis, which is Weyl's main focus in "Das Kontiuum", sets of natural numbers are extensions of properties built up step-by-step from the natural numbers by repeated application of the logical operations, with the crucial restriction of quantification to the domain of the natural numbers. Weyl calls this process of set-formation "the mathematical process" and contrasts his predicative concept of set with the dominant concept of set. He writes [73, p. 20]:

Finite sets can be described in two ways: either in individual terms, by exhibiting each of their elements, or in general terms, on the basis of a rule, i.e., by indicating properties which apply to the elements of the set and to no other objects. In the case of infinite sets, the first

\footnotetext{
${ }^{29}$ See $[57,58]$. See also [38, p. 378]. Note that I am here interested in the main ideas underlying this notion, rather than in an exegesis of Poincaré's thought.

${ }^{30}$ See also [12] for discussion.
} 
way is impossible (and this is the very essence of the infinite).

This brings Weyl to reject the meaningfulness of the powerset of an infinite set, including the set of all subsets of the natural numbers, as it is not amenable to a general description in terms of rules. The openendedness of infinite sets means that infinite subsets of the natural numbers should be defined by rules which specify properties of the natural numbers. Weyl [73, p. 23] writes:

The representation of an infinite set as a "gathering" brought together by infinitely many individual arbitrary acts of selection, assembled, and then surveyed as a whole by consciousness, is nonsensical; "inexhaustibility" is essential to the infinite. [...] Therefore I contrast the concept of set and function formulated here in an exact way with the completely vague concept of function which has become canonical in analysis since Dirichlet and, together with it, the prevailing concept of set.

A remarkable aspect of Weyl's concept of set is the inductive generation of the properties of the natural numbers through iterated application of the logical operations (with restricted quantifiers). Weyl lucidly highlights the crucial role of this iteration for the mathematical process. I will get back to this at the end of Section 4.3.3.

I have emphasised two significant aspects in Weyl's "Das Kontinuum": the objection to the powerset of an infinite set and the role of a potentialist view of infinity. It is interesting to compare these with more recent discussions on predicativity. The impredicativity and the arbitrariness of the powerset of an infinite set is also exposed in a fundamental article by [48], in which the author sets out the details of a constructive set theory that, notwithstanding its use of intuitionistic logic, bears strong formal affinities with ZF set theory. Myhill replaces the powerset axiom of ZF with a constructively weaker axiom of exponentiation, as the first is seen as lacking constructive justification. ${ }^{31}$ Myhill's criticism of the powerset axiom of ZF is particularly clear, and deserves quoting:

Power set seems especially nonconstructive and impredicative compared with the other axioms [of set theory]: it does not involve, as the others do, putting together or taking apart sets that one has already constructed but rather selecting out of the totality of all sets, all those

\footnotetext{
${ }^{31}$ The axiom of exponentiation allows us to collect in a set all the functions from a set $A$ to a set $B$. This is constructively weaker than the full powerset [48, 2].
} 
that stand in the relation of inclusion with a given set.

[48, p. 351]

We have here the opposition between, on the one side, the arbitrariness of the powerset of an infinite set, whose justification seems to require the prior availability or even surveyability of an infinite mathematical domain, with, on the other side, the rule-like construction of a set. This strongly resonates with the typical constructive appeal of inductive definitions, which has been repeatedly stressed above: the rule-like build up of a set from some initial unproblematic elements. We also saw that inductive definitions are often introduced today to eliminate problematic uses of the powerset of infinite sets, almost as if they were computationally approximating from below as much as possible of the powerset of an infinite set. Furthermore, the monontonicity of inductive definitions would seem to ensure that at no time the generation of new elements "disrupts" or modifies earlier fragments of the set - at least in the sense that what has entered the set at a certain stage cannot leave it at subsequent stages.

As to the role of potential infinity in Weyl's analysis, this also has a counterpart in more recent discussions. In the fundamental [42], the authors introduce (generalised) inductive definitions and argue for their constructivity. In their conclusion they write that the method of inductive definitions

exhausts those means of definition at present known which are acceptable from a standpoint which rejects the actual infinite. ${ }^{32}$

In view of the rule-like character and the monotonicity of inductive definitions, as well as these remarkable similarities with recent discussions on predicativity, it seems at least possible to give a predicative justification of these constructions along the lines of Poincarè and Weyl's considerations. The challenge here is to sharpen the notion of invariance in a way that more directly applies to the case of inductive definitions. ${ }^{33}$

\footnotetext{
[41].

${ }^{32}$ I would like to thank a referee for drawing my attention to this passage and to

${ }^{33} \mathrm{~A}$ thorough discussion of this point would require careful consideration of Lorenzen's work. See e.g. [41]. Note that one could argue that the term "predicativity" is now been used to refer to a different phenomenon altogether compared with that giving rise to the $\Gamma_{0}$ limit. This seems to be Feferman's point of view in [26, p. 4-5], when discussing especially Lorenzen and Wang's work on predicativity. I am persuaded this is a complex issue that would require careful consideration.
} 
4.3.2. Logic. The constructivist could explore another, not necessarily disjoint, strategy, by pursuing the question whether the underlying logic has a role to play in an assessment of the predicativity of inductive definitions. More specifically, one could argue that a shift to intuitionistic logic makes a defense of the predicativity of inductive definitions more plausible.

The idea is to extend to the case of generalised inductive definitions considerations that arise for the inductive definition of the natural numbers. Elsewhere, I analyse the presuppositions of a predicativist argument for intuitionistic logic inspired by Dummett's argument for indefinite extensibility [20]. ${ }^{34}$ This argument makes essential use of a claim that mathematical induction involves a form of circularity. ${ }^{35}$ In section 4.1, we saw the inductive definition of the natural numbers as the least set containing 0 and closed with respect to the successor operation. The closure condition for the natural numbers is expressed by the principle of mathematical induction. Mathematical induction is a fundamental principle in arithmetic, which enables us to prove universal statements as follows: it suffices to show that a property, say $F$, holds of the first natural number, 0 , and that it progresses from a number to the next one, i.e. that if $F$ holds of $n$, it also holds of $\operatorname{suc}(n)$. Then we can conclude that $F$ holds of every natural number.

Though the natural numbers are considered unproblematic according to both forms of predicativity under examination here, one may claim that a thorough predicativist perspective should recognize the impredicativity of the principle of mathematical induction [53]. The worry regarding induction is that this minimality condition involves a circularity. One way of expressing this concern is by observing that the principle of mathematical induction is stated for arbitrary properties. Therefore, it also applies to those properties, $F$, that refer to the whole set of natural numbers. In other terms, the formula which describes the property $F$ in the principle of mathematical induction may contain unrestricted number quantifiers, like, for example, a universal quantifier ranging over all the natural numbers. The set of natural numbers would then be defined by reference to the whole natural numbers, against the $\mathrm{VCP}{ }^{36}$

The thought scrutinised in [20] is that while standard interpretations of classical quantification require the availability of each element of the domain prior to quantification and therefore give rise to the difficulties

\footnotetext{
${ }^{34}$ See also $[39,40]$.

${ }^{35}$ See $[22,49,53]$.

${ }^{36}$ See $[49,53]$. See also $[17,20]$ for a detailed analysis of the natural number case.
} 
above, an intuitionistic universal quantifier may by given a generic interpretation. In particular, the universal quantifiers ranging over the natural numbers that we find in the principle of mathematical induction can be given a generic interpretation in an intuitionistic context. This would seem to suffice to eliminate the difficulty involved with the circularity of mathematical induction. The constructivist could hope that considerations such as these could be extended to the case of generalised inductive definitions, so to ease the difficulty with the apparent circularity of the closure condition which was discussed in section 4.2.

4.3.3. Trees. In Section 4.2, we saw that a generalised inductive definition can be presented in stages, indexed by classical (impredicative) ordinals. The constructivist could employ well-founded trees within an intuistionistic context to play a role analogous to that of the classical ordinals in the classical context and argue that, constructively, well-founded trees are directly predicatively justified. This seems to be a view often put forth by constructive type theorists. For example, [52] compares predicativity given the natural numbers with "the constructivist notion of predicativity which recognises a construction as predicative if it has a clear inductive structure, e.g. W-sets and superuniverses." 37 A constructivist who wished to proceed along this route, would need to explain what grants, in an intuitionistic context, a direct justification of the induction principles that express the closure of (at least some) generalised inductive definition. Perhaps, one could proceed by analogy to the case of predicativity given the natural numbers. That form of predicativity takes the natural numbers as given, and in so doing accepts as unproblematic the principle of mathematical induction. One could perhaps make a similar move in the case of inductive definitions, claiming that the relevant form of transfinite induction is predicatively justified at least on the basis of intuitionistic logic. An argument along these lines could also employ some of the considerations from the previous subsection, as one may insist that the intuitionistic focus on proofs rather than objects could make such an assumption more acceptable. Once more, Poincaré and Weyl's philosophies of mathematics could be source of inspiration. Both mathematicians insisted on the impossibility of giving a reduction of the principle of mathematical induction. Poincaré appealed to a form of intuition to justify it. Weyl made very clear the crucial role of the principle of iteration within his predicative foundation of analysis and in mathematics more generally. One could then explore the plausibility of an approach to predicativity which takes the inductive definition of the

\footnotetext{
${ }^{37}$ See also $[24,25]$.
} 
natural numbers as paradigmatic example of more general forms of inductive definitions that are taken as given and no further reducible.

\section{Conclusion}

In this note, I have offered motivation, stemming from the current mathematical practice, for an investigation into the notion of predicativity and especially constructive predicativity. I have highlighted the role of predicativity in current debates, and its key role in concrete practical applications, where it acts as a criteria for the correctness of computation and for consistency. Inductive definitions represent powerful expressive means of definition, which are increasingly employed in the constructive practice. In that context, they are usually considered justified not only from a constructive but also from a predicative point of view. The predicative justification of inductive definitions, though, requires further thought. One of the points of concern is the fact that these definitions are impredicative according to the prooftheoretic analysis of predicativity put forth by Kreisel, Feferman and Schütte. More specifically, the proof-theoretic strength of theories of inductive definitions exceeds by far the proof-theoretic strength of theories which are recognised as predicative according to that analysis. This leaves open the question of what could be taken to offer predicative justification to inductive definitions from a constructive perspective and, therefore, what characterises constructive predicativity. I have offered three (non-exclusive) suggestions. One would be to explore the original debates on predicativity, especially Poincaré and Weyl's contributions, as they present us with ideas which have significant affinities with those emerging in more recent debates. Another option is to focus on the role of different understanding of quantification, and explore whether a shift to intuitionistic rather than classical logic could eliminate or alleviate the perceived difficulties with inductive definitions. Finally, the third option is to explore the role of the paradigmatic example of the natural numbers, with its principle of induction, for a new constructive route to a justification of the stratification in stages of an inductively defined

set. Here the principal question is what could grant the constructivist's belief that the relevant well-founded trees are constructively and predicatively acceptable.

\section{REFERENCES}

[1] P. Aczel. An introduction to inductive definitions. In Jon Barwise, editor, Handbook of Mathematical Logic, volume 90 of Studies in Logic and the Foundations of Mathematics, pages 739 - 782. Elsevier, 1977. 
[2] P. Aczel. The type theoretic interpretation of constructive set theory. In A. MacIntyre, L. Pacholski, and J. Paris, editors, Logic Colloquium 'r7, pages 55-66. North-Holland, Amsterdam-New York, 1978.

[3] P. Aczel. The type theoretic interpretation of constructive set theory: Inductive definitions. In R. B. Marcus, G. J. Dorn, and G. J. W. Dorn, editors, Logic, Methodology, and Philosophy of Science VII, pages 17-49. North-Holland, Amsterdam and New York, 1986.

[4] AGDA. Agda wiki, 2020. Available at http://wiki.portal.chalmers.se/agda/pmwiki.php.

[5] F. Barbanera and S. Berardi. Proof-irrelevance out of excluded-middle and choice in the calculus of constructions. Journal of Functional Programming, 6(3):519-525, 1996.

[6] J. Barwise. Admissible sets and structures. An approach to definability theory. Springer Verlag, Berlin, 1975.

[7] M. Beeson. Foundations of Constructive Mathematics. Springer Verlag, Berlin, 1985.

[8] P. Benacerraf and H. Putnam. Philosophy of Mathematics: Selected Readings. Cambridge University Press, 1983.

[9] E. Bishop. Foundations of constructive analysis. McGraw-Hill, New York, 1967.

[10] D. S. Bridges and F. Richman. Varieties of Constructive Mathematics. Cambridge University Press, 1987.

[11] W. Buchholz, S. Feferman, W. Pohlers, and W. Sieg. Iterated inductive definitions and subsystems of analysis. Springer, Berlin, 1981.

[12] A. Cantini. Truth and the philosophy of mathematics. This volume, 2020.

[13] R. L. Constable and et al. Implementing Mathematics with the Nuprl Proof Development System. Prentice-Hall, Englewood Cliffs, New Jersey, 1986.

[14] T. Coquand. Metamathematical investigations of a calculus of constructions. Technical report, INRIA, 1989.

[15] T. Coquand and G. Huet. The calculus of constructions. Technical Report RR-0530, INRIA, May 1986.

[16] T. Coquand, G. Sambin, J. Smith, and S. Valentini. Inductively generated formal topologies. Annals of Pure and Applied Logic, 124(1):71 - 106, 2003.

[17] L. Crosilla. Constructivity and Predicativity: Philosophical foundations. PhD thesis, School of Philosophy, Religion and the History of Science, University of Leeds, 2016.

[18] L. Crosilla. Predicativity and Feferman. In G. Jäger and W. Sieg, editors, Feferman on Foundations: Logic, Mathematics, Philosophy, Outstanding Contributions to Logic. Springer, 2017. Forthcoming.

[19] L. Crosilla. The entanglement of logic and set theory, constructively. Inquiry, $0(0): 1-22,2019$.

[20] L. Crosilla. From predicativity to intuitionistic mathematics, via Dummett. Unpublished Manuscript, 2020.

[21] W. Dean and S. Walsh. The prehistory of the subsystems of second-order arithmetic. Rev. Symb. Log., 10:357-396, 2016.

[22] M. Dummett. The Philosophical Significance of Gödel's Theorem. Ratio, 5:140155, 1963.

[23] P. Dybjer. A general formulation of simultaneous inductive-recursive definitions in type theory. The Journal of Symbolic Logic, 65(2):525-549, 2000. 
[24] P. Dybjer. Program Testing and The Meaning Explanations of Martin-Löf Type Theory. In P. Dybjer, S. Lindström, E. Palmgren, and B.G. Sundholm, editors, Epistemology versus Ontology, Essays on the Philosophy and Foundations of Mathematics in Honour of Per Martin-Löf, 2012.

[25] P. Dybjer and A. Setzer. Induction-recursion and initial algebras. Annals of Pure and Applied Logic, 124(1):1 - 47, 2003.

[26] S. Feferman. Systems of predicative analysis. Journal of Symbolic Logic, 29:130, 1964.

[27] S. Feferman. A language and axioms for explicit mathematics. In J. Crossley, editor, Algebra and Logic, volume 450 of Lecture Notes in Mathematics, pages 87-139. Springer, Berlin, 1975.

[28] S. Feferman. Weyl vindicated: Das Kontinuum seventy years later. In C. Cellucci and G. Sambin, editors, Temi e prospettive della logica e della scienza contemporanee, pages 59-93, 1988.

[29] S. Feferman. Comments on 'Predicativity as a philosophical position' by G. Hellman. Review Internationale de Philosophie, 229(3), 2004.

[30] S. Feferman. Predicativity. In S. Shapiro, editor, Handbook of the Philosophy of Mathematics and Logic. Oxford University Press, Oxford, 2005.

[31] S. Feferman. The proof theory of classical and constructive inductive definitions. a forty year saga, 1968 - 2008. In R. Schindler, editor, Ways of Proof Theory, pages 7-30. De Gruyter, 2013.

[32] S. Feferman. Why a little bit goes a long way: predicative foundations of analysis. Unpublished notes dating from 1977-1981, with a new introduction. Retrieved from the address: https://math.stanford.edu/ feferman/papers.html, 2013.

[33] H. Friedman. The consistency of classical set theory relative to a set theory with intuitionistic logic. Journal of Symbolic Logic, 38:315-319, 1973.

[34] J.Y. Girard. Interprétation fonctionnelle et élimination des coupures de l'arithmétique d'ordre supérieur. PhD thesis, These d'Etat, Paris VII, 1972.

[35] K. Gödel. Russell's mathematical logic. In P. A. Schlipp, editor, The philosophy of Bertrand Russell, pages 123-153. Northwestern University, Evanston and Chicago, 1944. Reprinted in [8]. (Page references are to the reprinting).

[36] G. Gonthier. Formal Proof-The Four-Color Theorem. Notices of the American Mathematical Society, 11(55):1382-1393, 2008.

[37] G. Kreisel. Ordinal logics and the characterization of informal concepts of proof. In Proceedings of the International Congress of Mathematicians (August 1958), pages 289-299. Gauthier-Villars, Paris, 1958.

[38] G. Kreisel. La prédicativité. Bulletin de la Societé Mathématique de France, 88:371-391, 1960.

[39] Ø. Linnebo. Dummett on indefinite extensibility. Philosophical Issues, 28(1):196-220, 2018.

[40] Ø. Linnebo. Generality explained, 2018. Unpublished manuscript.

[41] P. Lorenzen. Logical reflection and formalism. The Journal of Symbolic Logic, 23(3):241-249, 1958.

[42] P. Lorenzen and J. Myhill. Constructive definition of certain analytic sets of numbers. Journal of Symbolic Logic, 24:37-49, 1959. 
[43] P. Martin-Löf. An intuitionistic theory of types: predicative part. In H. E. Rose and J. C. Shepherdson, editors, Logic Colloquium 1973. North-Holland, Amsterdam, 1975.

[44] P. Martin-Löf. Constructive mathematics and computer programming. In L. J. Choen, editor, Logic, Methodology, and Philosophy of Science VI. NorthHolland, Amsterdam, 1982.

[45] P. Martin-Löf. Intuitionistic Type Theory. Bibliopolis, Naples, 1984.

[46] P. Martin-Löf. The Hilbert-Brouwer controversy resolved? In et al. van Atten, editor, One Hundred Years of Intuitionism (1907 - 2007), pages 243-256. Publications des Archives Henri Poincaré, 2008.

[47] Y.N. Moschovakis. Elementary Induction on Abstract Structures (Studies in Logic and the Foundations of Mathematics). American Elsevier Pub. Co, 1974.

[48] J. Myhill. Constructive set theory. Journal of Symbolic Logic, 40:347-382, 1975.

[49] E. Nelson. Predicative arithmetic. Princeton University Press, Princeton, 1986.

[50] B. Nordström, K. Petersson, and J. M. Smith. Programming in Martin-Löf's Type Theory: an introduction. Clarendon Press, 1990.

[51] E. Palmgren. Type-theoretic interpretation of iterated, strictly positive inductive definitions. Arch Math Logic, 32:75-99, 1992.

[52] E. Palmgren. On universes in type theory. In G. Sambin and J. Smith, editors, Twenty-five years of type theory. Oxford University Press, Oxford, 1998.

[53] C. Parsons. The impredicativity of induction. In M. Detlefsen, editor, Proof, Logic, and Formalization, pages 139-161. Routledge, London, 1992.

[54] H. Poincaré. Les mathématiques et la logique. Revue de Métaphysique et Morale, 1:815-835, 1905.

[55] H. Poincaré. Les mathématiques et la logique. Revue de Métaphysique et de Morale, 2:17-34, 1906.

[56] H. Poincaré. Les mathématiques et la logique. Revue de Métaphysique et de Morale, 14:294-317, 1906.

[57] H. Poincaré. La logique de l'infini. Revue de Métaphysique et Morale, 17:461482, 1909.

[58] H. Poincaré. La logique de l'infini. Scientia, 12:1-11, 1912.

[59] M. Rathjen. The constructive Hilbert program and the limits of Martin-Löf type theory. Synthese, 147:81-120, 2005.

[60] M. Rathjen, E. Griffor, and E. Palmgren. Inaccessibility in constructive set theory and type theory. Annals of Pure and Applied Logic, 94:181-200, 1998.

[61] B. Russell. Les paradoxes de la logique. Revue de métaphysique et de morale, 14:627-650, 1906.

[62] B. Russell. On Some Difficulties in the Theory of Transfinite Numbers and Order Types. Proceedings of the London Mathematical Society, 4:29-53, 1906.

[63] B. Russell. Mathematical logic as based on the theory of types. American Journal of Mathematics, 30:222-262, 1908.

[64] B. Russell. Essays in Analysis. George Braziller, New York, 1973. Edited by D. Lackey.

[65] G. Sambin. Intuitionistic formal spaces - a first communication. In D. Skordev, editor, Mathematical Logic and its Applications, pages 187-204. Plenum, 1987.

[66] K. Schütte. Eine Grenze für die Beweisbarkeit der Transfiniten Induktion in der verzweigten Typenlogik. Archiv für mathematische Logik und Grundlagenforschung, 7:45-60, 1965. 
[67] K. Schütte. Predicative well-orderings. In J. Crossley and M. Dummett, editors, Formal Systems and Recursive Functions. North-Holland, Amsterdam, 1965.

[68] S. G. Simpson. Partial realizations of Hilbert's program. Journal of Symbolic Logic, 53(2):349-363, 1988.

[69] S. G. Simpson. Subsystems of Second Order Arithmetic. Perspectives in Mathematical Logic. Springer-Verlag, 1999.

[70] The Coq Development Team. Coq, 2020. https://coq.inria.fr.

[71] The Univalent Foundations Program. Homotopy Type Theory: Univalent Foundations of Mathematics. Institute of Advanced Studies, 2013.

[72] H. Wang. Ordinal numbers and predicative set theory. Zeitschr. f. math.Logik und Grundlagen d. Math., 5:pp. 216-239, 1959.

[73] H. Weyl. Das Kontinuum. Kritische Untersuchungen über die Grundlagen der Analysis. Veit, Leipzig, 1918.

[74] A. N. Whitehead and B. Russell. Principia Mathematica, 3 Vols., volume 1. Cambridge: Cambridge University Press, 1910, 1912, 1913. Second edition, 1925 (Vol 1), 1927 (Vols 2, 3); abridged as Principia Mathematica to *56, Cambridge: Cambridge University Press, 1962.

University of Oslo, E-MAIL: LAURA.Crosilla@IFIKK.UIO.NO 